\title{
SMART SIMULATION FOR DECISION SUPPORT AT HEADQUARTERS
}

\author{
Ariane BITOUN, Hans ten BERGEN, Yann PRUDENT \\ MASA Group, Paris, France \\ ariane.bitoun@masagroup.net, hans.tenbergen@masagroup.net, \\ yann.prudent@masagroup.net
}

\begin{abstract}
While serious games are being widely adopted by NATO and partner nations, their use is currently limited to training and operations planning. In this paper, we explore new methods that use simulations for decision support during the execution of military operations. During this phase, the commander makes decisions based on knowledge of the situation and the primary objectives. We propose here to take a simulation containing smart and autonomous units, and use it to create new kinds of decision support tools capable of improving situation awareness, and consequently the quality of decisions. The breakthrough behind this initiative is the realization that we can provide $H Q$ decision makers with access to a version of the information that smart simulated units use to make decisions. To ensure the approach was sound we first studied decision-making processes, and analyzed how situation awareness improves decision-making. After analysis of the decision-making processes at various headquarters, and the types of decision criteria employed, we are able to produce innovative information, computed by the simulation, and fed by the command and control system. We then propose a prerequisite architecture and describe the first results of our proof of concept work based on the SWORD (Simulation War gaming for Operational Research and Doctrine) simulation.
\end{abstract}

\section{Keywords: simulation, Headquarters, decision support, artificial intelligence}

\section{Simulation and Military Decisions Support Systems}

\subsection{Improving Decision Support by Resolving Information Overload}

To identify the skills that are necessary for military decision-making, we first investigated the underlying decision processes, and identified the features required for an ideal decision support system.

\subsubsection{A decision process model}

A generic decision process for organizations was first proposed by Simon (1965) [1], and extended by Mora et al. (2003). It consists of five consecutive phases: intelligence, design, choice, implementation and learning [2]. These decision phases can further be divided into several decision steps. (Figure 1)

\begin{tabular}{|l|l|}
\hline \multicolumn{1}{|c|}{ Decision Phase } & \multicolumn{1}{c|}{ Decision Step } \\
\hline \multirow{2}{*}{ Intelligence } & Data Gathering \\
\cline { 2 - 2 } & Problem Recognition \\
\hline \multirow{2}{*}{ Design } & Model Formulation \\
\cline { 2 - 2 } & Model Analysis \\
\hline \multirow{2}{*}{ Choice } & Evaluation \\
\cline { 2 - 2 } & Selection \\
\hline \multirow{4}{*}{ Implementation } & Presentation of Results \\
\cline { 2 - 2 } & Task Planning \\
\cline { 2 - 2 } & Task Tracking \\
\hline \multirow{2}{*}{ Learning } & Outcome-Process Link Analysis \\
\cline { 2 - 2 } & Outcome-Process Link Synthesis \\
\hline
\end{tabular}

Figure 1: The generic decision process presented by Mora et al.(2003)

During the intelligence phase, decision makers observe reality, gather information 
and therefore gain an understanding of the problems and possibilities at hand [3]. In the design phase, they construct a decision model, which consists of possible actions, decision criteria, uncontrollable events and relationships between these variables. This decision model serves as a basis for the choice phase, in which decision makers evaluate possible alternatives and generate recommended actions. In the implementation phase, decision makers weigh possible consequences and gain confidence about their decision, plan the tasks needed to realize the decision and execute this plan.

In order to improve their decision behavior, as well as assessing decision situations, decision makers observe the outcomes of the decision and connect it to their decision behavior in the learning phase.

1.1.2 Supporting the decision by enhancing situation awareness

In her model, Endsley (1995) [4] presents situation awareness as a stage separate from decision-making and action. Situation awareness is described as the decisionmaker's internal model of the state of the environment. Based on that representation, the decision-maker can decide what to do about the situation and carry out any necessary actions. There is thus a strong link between situation awareness and the decision-making process. Situation awareness is represented as the main precursor to decision-making, and is the key factor determining decision quality.

Clearly, circumstances where no information is available should result in poor situation awareness, leading with high probability to very low decision quality. One could even claim that a good approach to reach optimal situation awareness would be to provide as much information as possible. However, this does not necessarily represent the best solution, as more information does not automatically mean better situation awareness to ensure better human performance. First, all this information may exceed the human information processing capabilities, leading to cognitive overload. Second, not all the data and information available in the environment is relevant and useful for reaching an optimal decision

In fact, in some situations, most of the data can be seen as distracters and noise for the decision-maker, and may thus reduce his/her level of situation awareness.

The decision-maker must detect and use only a specific fraction of this information to enhance his/her decision making processes. Such considerations lead to the concept of "the right information, at the right place, at the right time", as opposed to "all information, everywhere, all the time". This is illustrated in the figure 2 .

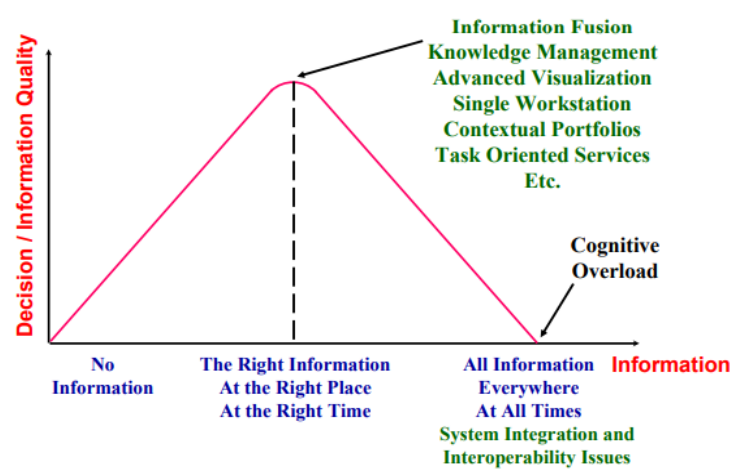

Figure 2: Decision and information quality

\subsubsection{Theoretical demand for intelligent filtering of information}

According to Auger et al (2006) [5] and its definition of an ideal support system, such a tool must be able to:

- provide the information needed by the various human decision-makers, and complement the cognitive power of the human mind by proposing a powerful and operational fusion of data

- provide a system that can be controlled effortlessly by a human by producing an intuitive representation adapted to each operator's specialty (artillery, synthesis, communication, etc).

- support a wide variety of problemsolving strategy evaluations by proposing a situational analysis of the battlefield and a simulation of alternative evolutions and maneuvers. 


\subsection{Existing Use of Simulation for Military Decision Support}

Today simulations are mainly used for military decision support in two cases: force generation and war gaming.

- Force generation: Consists of listing the tasks to be performed, deducing the capacities needed by operational functions and then placing the tasks to be performed in a space-time framework. Modern-day computing power can be used to simulate several scenarios in rapid succession, which increases the overall time available for decision makers.

- War gaming: In the context of existing methods available for operation planning, competition between friendly course of action and enemy probable course of action, confirms a preference for a course of action.

In short, we can conclude that the traditional uses of simulation for decision support concentrate on running scenarios, which aims to improve strategy conception and planning.

\subsection{Proposal: an Improved} Understanding of the Battlefield Using a Smart Synthesis of Field Information

In a typical multi-agent simulation, the situation representation layer, also termed the 'low level AI' layer (Figure 3), is traditionally used by the simulated agent to make sense of raw data from the simulation, allowing the agent to make the best possible decisions and take the right actions. Our idea consists of going beyond the traditional use of a simulation by providing the officers at headquarters access to the situation representation layer, which improves their understanding of the current position in the field.

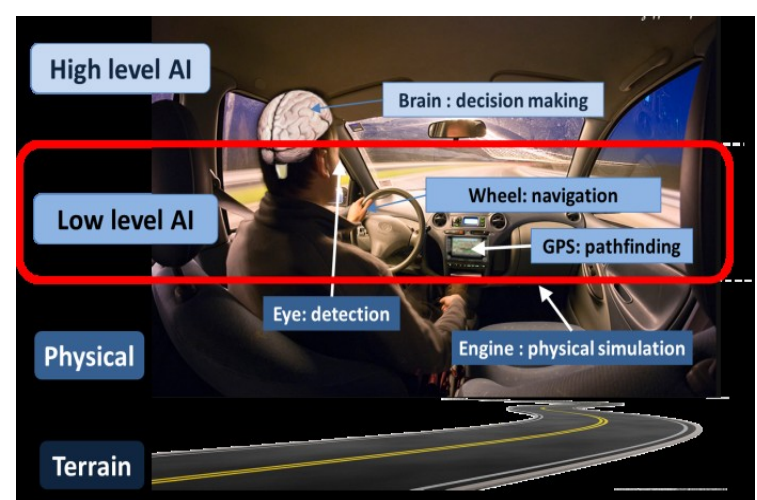

Figure 3: Simulation modules

The SWORD simulation for proof of concept. To achieve our aims we decided to consider the SWORD simulation - currently in use in many armies - as it is a multiagent simulation, which calculates a battlefield representation for each simulated agent, allowing each one to autonomously decide on their behavior, in accordance with their doctrine. We believe that we can offer HQ officers an innovative battlefield representation based on the one already used in SWORD's situation representation layer.

This information can be processed to give HQ officers enhanced information regarding the evaluation of forces' capacities, the danger enemies represent, force ratios, etc.

\section{Computed Battlefield Representation for Simulated Agents}

2.1 Features of Multi-Agent Systems: AI and Algorithms

As defined by P. Maess (1995) [6], "autonomous agents are computational systems that inhabit some complex dynamic environment, sense and act autonomously in this environment, and by doing so realize a set of goals or tasks for which they are designed". The word computational, when paired with agent, has a very different meaning from a biological agent, which includes humans, animals, and bacteria. Autonomous means that computational agents operate without the intervention of any entities, and have control over their actions. (Figure 4) 


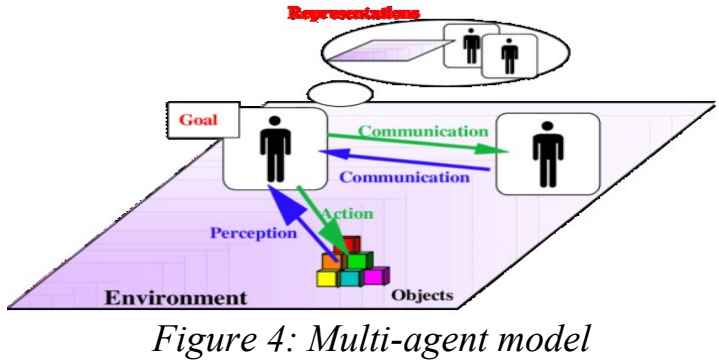

Assigning goals to an agent means that the said agent will act upon its environment in order to achieve a specified objective, and that in doing so the agent demonstrates a type of rational behavior, in the sense that it minimizes or maximizes its performance measured within its environment. Behavior here means the action that is performed after receiving sensory inputs (or any sequence of sensory inputs). In addition to the sensory inputs, actions, and goals an agent may include domain knowledge (knowledge of a particular environment or problem to be solved). This knowledge can be algorithmic, Artificial Intelligence (AI) based, heuristics, etc. In SWORD this knowledge is given by Direct AI technology.

\subsection{Main Principles of the Direct AI Technology}

In a nutshell, Direct AI is a technology for engineering an agent's brain, the part of the agent responsible for its actions in the application environment. More precisely, Direct AI provides a solution to the problem of action selection, that is, the problem of deciding what to do next. As we will see shortly, the way we reason, design, and express behaviors in Direct AI, is especially adequate when it comes to deciding on a course of action that is a compromise between many different, sometimes contradictory or conflicting, courses of action. A military Soldier may be confronted with a behavior that suggests a number of positions for observing an enemy, and a different behavior, a safeguarding instinct, that suggests adopting stealth positions to avoid taking unnecessary risks of being seen. A brain decides how to act on its application environment based on its perception and knowledge of its environment. We say the agent is situated in the environment, since its actions may not only change the environment in which it takes an active part, they may also have an effect on the agent itself, and perhaps on other agents. This, in turn, might have an effect on the agent's future decisions. An agent thinks and acts in a changing, unpredictable, environment. The fact that the agent is situated means that the agent has a partial and local perception of the environment; it can only sense the environment around it, and may not know everything there is to know about it, just what it can learn about it.

\subsection{SWORD Battlefield Representation}

SWORD is a constructive simulation that is natively used to immerse brigade and division command post staff in large-scale conflicts, stabilization operations, terrorist threats or natural disasters. (Figure 5) SWORD simulates a diverse range of situations in realistic environments and, powered by Direct AI, lets trainees lead thousands of autonomous subordinate units (at platoon and company levels) on the virtual field. Agents can receive operation orders and execute them without additional input from the players, while adapting their behavior accordingly as the situation evolves.

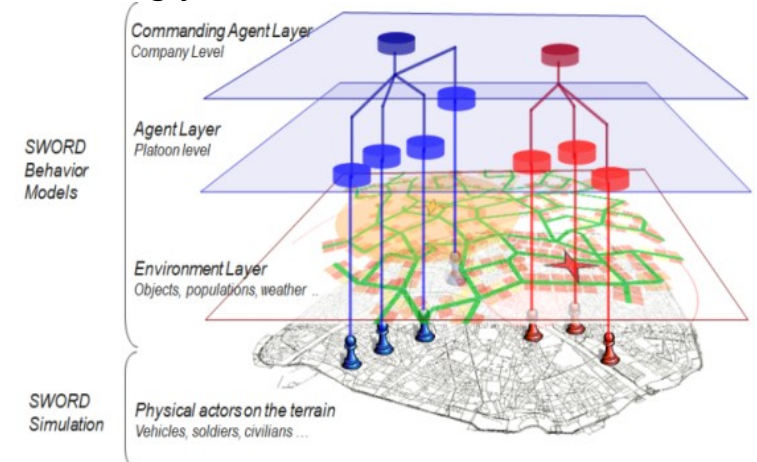

Figure 5: SWORD layers

Their behaviors are validated by doctrinal experts and end-users. Their model depends on algorithms that make agents perceive, move, communicate, shoot, as well as the capacities of underlying equipment, as 
described in a database. As military officers at HQ or in the field do, each unit makes autonomous decisions based on the doctrine that describes the expected course of action and the results of each mission, its current mission, its operational state, the tactical situation, the terrain, the time elapsed, etc.

\section{Making Sense of Data Through Smart Visualization and Processing}

\subsection{Methodology Used}

As discussed earlier, one of the effects of many information systems is a tendency for information overload, which invariably leads to a state of decision-making paralysis. Indeed, identifying and presenting only strictly relevant information to decision makers is key. As a rule, the volume of information used by the lead decision maker is surprisingly limited, immediately implying that a large part of the information collected has no impact on decision-making. (Figure 6) Information communicated to the lead decision maker must therefore be a high level summary. For instance, commanders of large numbers of units do not make decisions based on reports from hundreds of tanks, but rather on their perception of the enemy's movement, their overall understanding of a maneuver, the sum of the states of units, etc. Consequently there is a need to develop software that sorts, classifies, and synthesizes information.

Our first work consisted in defining which information is needed by which officer.

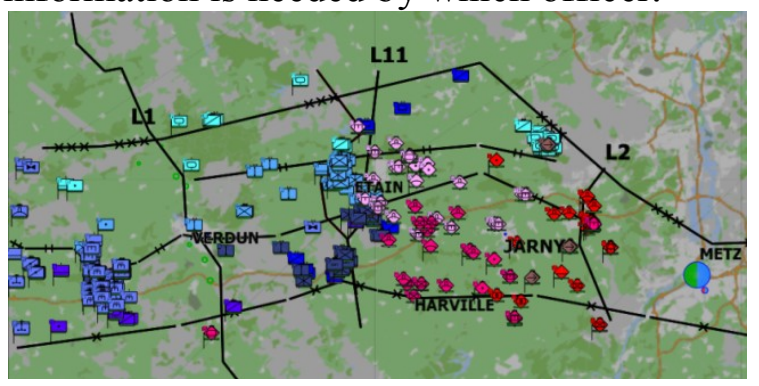

Figure 6: Example of complex tactical situation

This let us ask the following questions, for each cell officer: which information is needed? What is the expected, standard evolution of a situation? Conversely, when the unexpected occurs, which particular events would require an alert?

Our work is based on close collaboration with officers based at French army headquarters, whose approach is broadly similar to that of all major armies.

Together with military experts, and simultaneously consulting current doctrine, we attempted to analyze the type of innovative information that could be brought into command and control centers to improve their understanding of the situation and by extension help their decision-making processes.

We concluded that by combining reports from the field, algorithms, current status information, and AI, it is possible to offer an automated and dynamic calculation of a representation of the battlefield, and its possible evolution. The idea here not being to replace a command and control system, but rather to improve the situation awareness and to provide automatic warnings on specific events that require urgent investigation by the officer.

In this first implementation of a new approach to decision-making support, we suggest the following list of data that could feasibly be given to HQ officers in an effort to support the tactical situation synthesis, support/supply management, units in the field, and the conception of the next maneuver. The table below (Figure 7) allows you to follow the results of our analysis of user needs, classified by HQ cell.

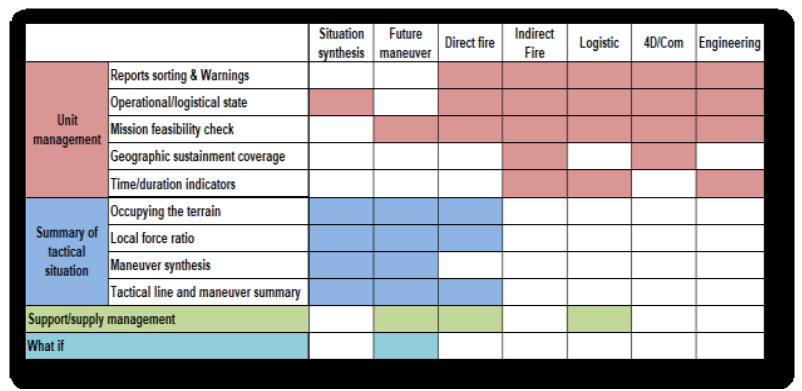

Figure 7: Analysis of smart date needs by $H Q$ cell

\subsection{Unit Management}

With the imminent arrival of the Scorpion program, that aims to modernize the French fleet with massive digitization, operators 
will receive a huge amount of reports from the battlefield. The next generation of command and control systems will need a filtering system indicating whether the reports generally show a normal evolution of the situation of the current mission, or, conversely, if units are encountering a specific event or difficulties on the battlefield. In order to help the management of units in the field, we propose an automatic filter and synthesis of the situation adapted to the specific needs of the operator. The purpose here is not to replace the traditional command and control system but to enhance it by alerting the HQ officer on specific events, offering a global summary of the state of units and synthesizing the state and the capacities of units.

The following are descriptions of functionality that this tool should cover.

3.2.1 Reports sorting \& automated warnings adapted to the context

The tool should automatically sort the reports depending on their importance. For example, the importance of detecting enemy units is not the same when done by units at the rear of the battlefield as when done by units during an attack mission.

The tool should also adapt the warning to the context. For example, calculating the time spent by units in specific zones, like $\mathrm{NRBC}$ or fire zones, and sending alarms to the operator when the units need to be replaced.

\subsubsection{Operational/Logistical state dynamic} calculation

Such a tool must be able to propose an automatic calculation of the global operational state of units, where major and other equipment are differentiated. In the same way, it must also be possible to propose a synthesis of the global logistical state of each unit. This information needs to be shown in a clear and simple way on the map, for instance with a life bar that describes the operational state of the unit and another bar to indicate the logistic status of the unit.

\subsubsection{Mission feasibility check}

Automatic calculation of resources needed for a mission, warning the operator whether the selected unit has enough resources to complete it. In a similar way, checking whether there is an open path to mission locations, warning the operator whenever a point cannot be reached.

\subsubsection{Dynamic geographic sustainment coverage}

For all the branches that provide a service (communication, indirect fire, etc.), the system must be able to calculate the geographic area covered by the service. The calculation depends on the terrain, the tactical situation, and remaining resources. For example, one will be able to show the geographic area covered by communication capacity, or the total area that artillery units can collectively attain, while factoring in the presence of major equipment, and the logistic status. As a result, this system will be able to alert a unit or specific geographic objective whether they are within a covered area.

\subsubsection{Times/Duration availability warnings}

Some specific units or missions, for example in artillery or engineering, need precise times for installation or preparation. Consequently, at a given moment, units can be available for action, while others are moving, installing, or packing. The system can also provide the time required for construction, the effects of drying, and schedule the availability of engineering units.

\subsubsection{Summary of tactical situation}

The purpose here is to provide the automatic generation of tactical maps. Using simulation algorithms, we can offer the automatic synthesis of the battlefield: the geographic ground occupation, the local force ratio, the global maneuver, the scheduled effects on the terrain and enemies. 


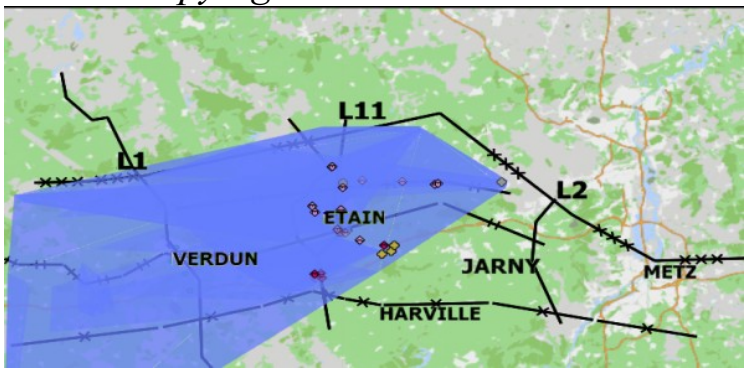

Figure 8: Terrain occupation

The system can calculate the total area occupied by all units, the entire perception zone based on available equipment, the current missions of units, and the potential area covered by fire. These calculations can be performed on the basis of the hierarchical level of units, their equipment capacities, current missions, and positions. Moreover, calculations for the density of forces could be added to provide an indication of the global force deployed at a glance.

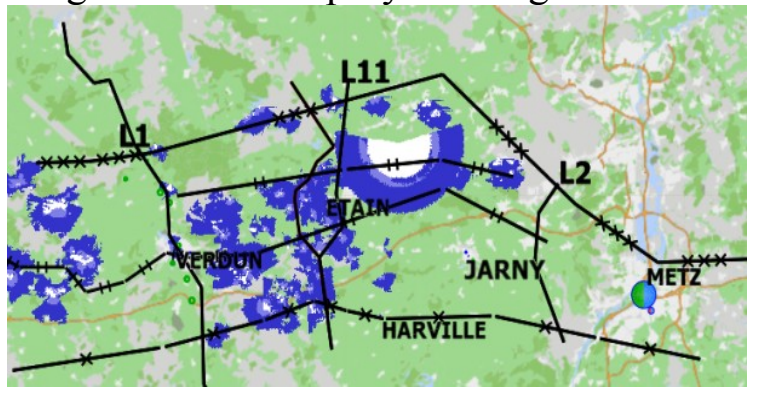

Figure 9: Perception capacities

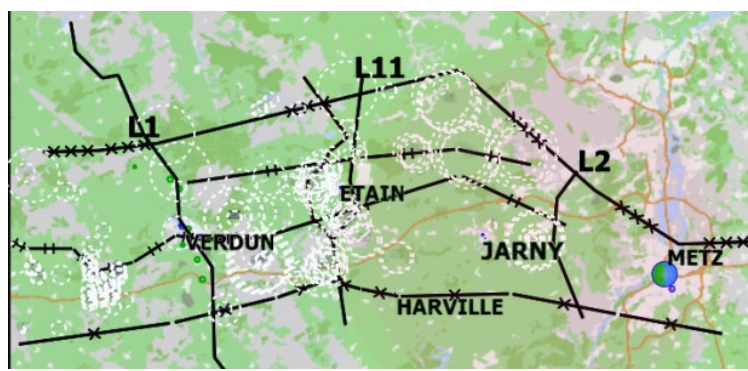

Figure 10: Direct fire capacities

\subsubsection{Local force ratio}

As seen earlier, the simulation calculates the force ratio of each agent, based on its knowledge of the tactical situation. It is therefore possible to offer a dedicated view of the local force ratios, which provides an insight into which forces, or area, may require reinforcements.

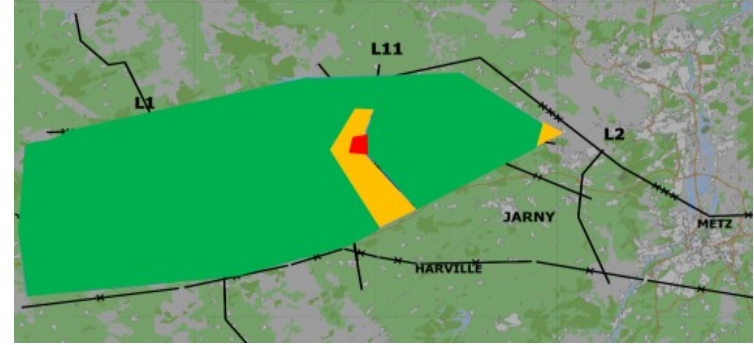

Figure 11: Local force ratio

\subsubsection{Tactical line generation and} maneuver summary

Based on current missions, knowledge of enemies, and capacities of units, we can generate a global maneuver summary, which includes a calculation of tactical lines, such as the Forward Line of Own Troops (FLOT), Limit Of Advance (LOA), Line of Contact (LC).

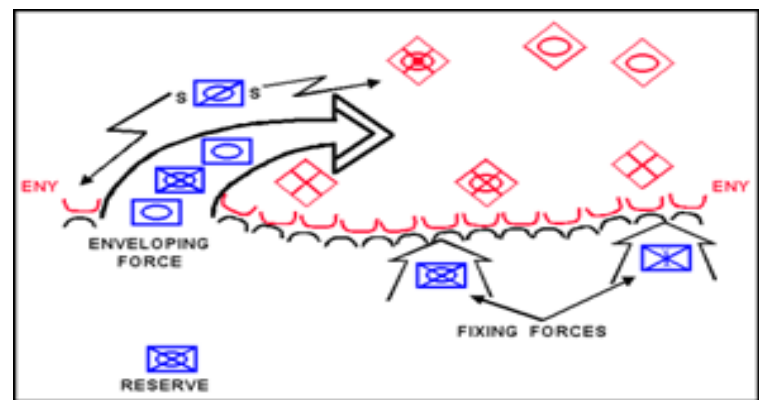

Figure 12: Maneuver summary

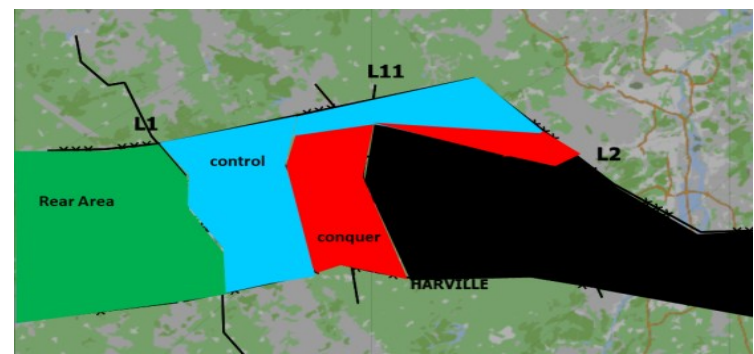

Figure 13: Expected effect summary

To take this analysis further, based on the expected effects approach, we could provide a maneuver view that relies on the major expected effects regarding the enemy and terrain. For example one could produce a map that differentiates between zones that must be recognized, conquered, controlled, etc., or enemies that must be eliminated or stopped. This could be achieved through the interpretation of the 
advancement of current missions, and the nature of planned missions.

\subsubsection{Support/Supply management}

For each unit on the battlefield, we provide a calculation of the time taken to be in a position to support a unit facing an enemy. To achieve this the simulation calculates the best route for each unit. This calculation takes into account all equipment capable of direct or indirect fire, known enemy positions, the terrain, friendly and enemy engineering works, tactical limits, etc. A filter could then offer a choice from logistic, direct or indirect fire support. The calculation can be reversed to automatically compute the time required to cover support units (medical, supply, etc.). This would include a means of calculating the time taken to reach all units that a medical supply unit is in charge of, and to generate warnings if the maximum time is exceeded.

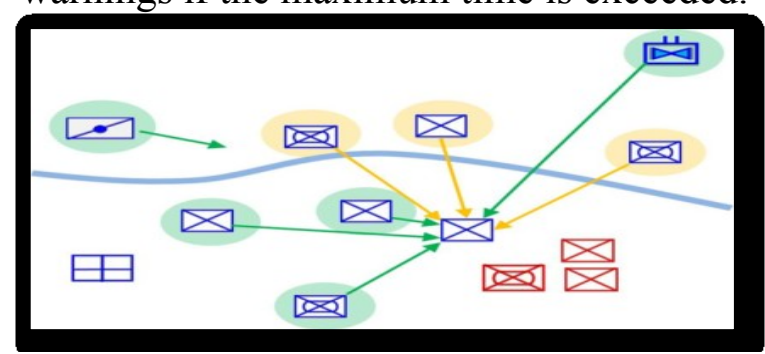

Figure 14: Fire support duration calculation for a unit

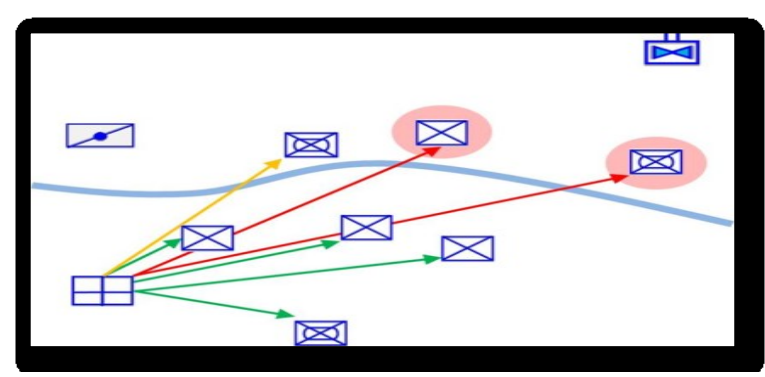

Figure 15: Support duration for supported units

\subsection{The "What if" Function}

As explained before, the wargaming use case is the most common use of a multiagent simulation as a decision support system at headquarters. The idea here is to offer an easy way to evaluate the current plan using the current knowledge of the enemy, or to simulate alternative options, hence "What if".

The main difficulties here are as follows:
- It must be simple for the user to give orders. Several options are available: prepared orders, very simple orders at a high level in the hierarchy (battalion, for example), graphical orders, etc.

- The user interface must be able to provide a bird's eye view of the situation that includes both friendly and enemy units. Furthermore, we must provide the option to give orders to enemy forces.

- The user must be able to modify the current situation, in order to view alternative, plausible situations. Examples include changing the order of battle, modifying the position and state of units, adjusting the enemy scenario, etc.

\section{Conclusion and Early Results 4.1 Recommended Prerequisite} Architecture

Here we outline a setup based on a multiagent battlefield representation, which lets us offer:

- Smart data visualization tools: raw data is processed by algorithms in the multiagent simulation, which gives them meaning and allows for immediate use through synthesis, alert generation, etc.

- Smart data exploitation tools: data can be the starting point for Artificial Intelligence processing, as it is in simulations or machine learning, with a view to help plan or evaluate next steps and future actions, or to go beyond this and help improve intelligence gathering or course of action analysis, etc.

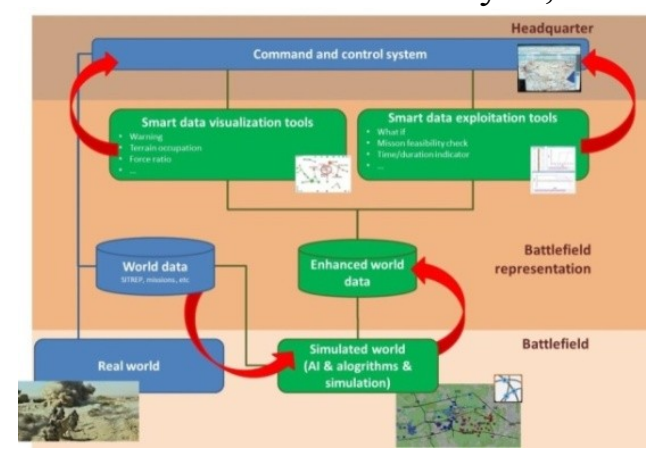

Figure 16: Architecture hypothesis 
All innovative methods proposed here depend on two principal prerequisites:

- a database containing all friendly equipment, plus presumed enemy equipment. Descriptions of all types of equipment must be accompanied by effect descriptions, to enable the simulation of the battlefield.

- the integration of the command and control systems within the tools described above, with a view to importing all data into the simulation: unit positions, logistic states, enemy knowledge, engineering work, NRBC zones, available missions, etc.

We then have to design a data representation that provides an easy-tounderstand, intuitive display of processed information. The last issue to be dealt with is the nature of the add-on tools we offer: should they be integrated into specific command and control systems, or be separate, mobile devices, such as tablets.

\subsection{Status Report and First Results}

We have already enriched SWORD functionality by making it possible to send queries that can retrieve information concerning the internal representation of the simulation. Development of data exploitation aspects has already begun, an example of which is shown on the right. Another example is shown earlier in the diagram labelled Terrain occupation. Early developments aimed at tactical situation synthesis and service coverage have already proved that the idea is valid, and that the SWORD simulation is ideally suited as a candidate for proof of concept, and will allow us to reap the benefits of this innovative approach.

All work will be evaluated during training sessions with the military, who will be the first to examine this alternative display, whose primary aim is to enhance situation awareness.

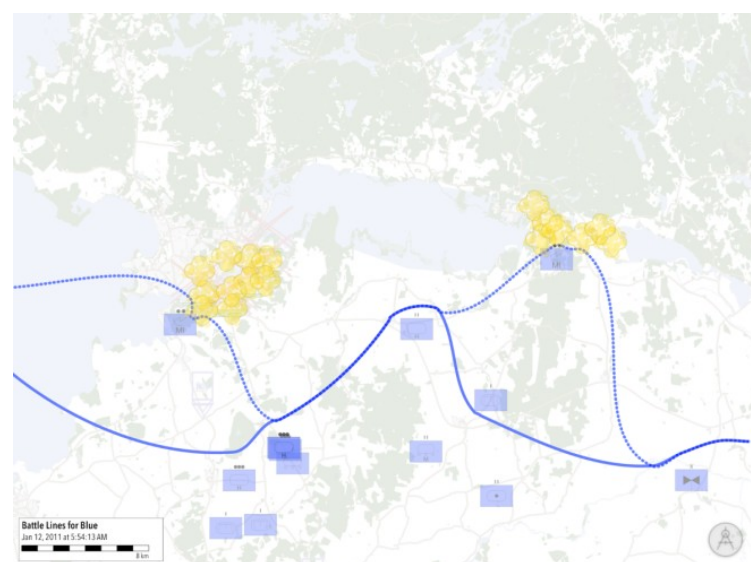

Figure 17: Example of battle lines generated automatically

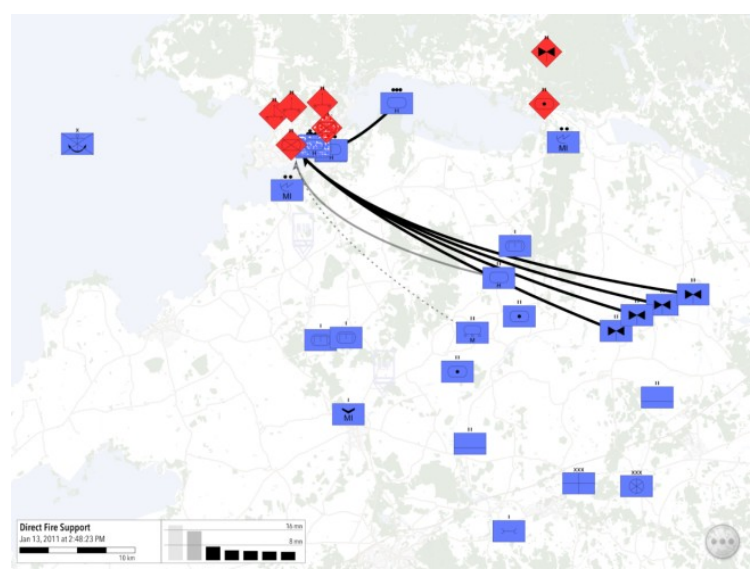

Figure 18: Example of automatic calculation of fire support duration

\subsection{Key Takeaways}

- The digitization of the army will impact decision processes at every level and officers will need next-generation tools to enhance their situation awareness.

- The use of the low-level AI layer and constructive simulation (combined with enhanced equipment and unit databases) connected to real data directly from the field can produce innovative information and help the work of officers.

- This new tool can provide a smart synthesis of the tactical situation, smart alerts according to the context and the current maneuver, smart calculations of realistic timeframes and geographic scope capacities. 


\section{Acknowledgements}

This paper is based on the STRATEGIC research project funded by the Direction
Générale de l'Armement (DGA) through the ASTRID Maturation program.

\section{References}

[1] Simon H. A, Administrative behavior, Cambridge UnivPress, 1965.

[2] Mora M., Forgionne G., Gupta J., Cervantes F., \& Gelman O., A framework to assess intelligent decision-making support systems. In: Knowledge-Based Intelligent Information and Engineering Systems, p. 63, 2003.

[3] Mora M., Forgionne G., Gupta J., Cervantes F., \& Gelman O., A framework to assess intelligent decision-making support systems. In: Knowledge-Based Intelligent Information and Engineering Systems, pp. 59-60, 2003.

[4] Endsley M. R., "Toward a Theory of Situation Awareness in Dynamic Systems", In: Human Factors 37.1, 32-64, 1995.

[5] Auger, Gouin, Roy \& DRDC Valcartier, Decision Support and knowledge exploitation technologies for C4ISR, 2006.

[6] Maess P., Artificial life meets entertainment: Life like autonomous agents, Communications of the ACM, Vol. 38, No. 11, pp. 108-114, 1995.

[7] Boyd, John, R., The Essence of Winning, and Losing, 28 June 1995, available at http://www.danford.net/boyd/essence.htm.

[8] Chen Miao, Zhu Qiuming and Chen Zhengxin, An Integrated Interactive Environment for Knowledge Discovery from Heterogeneous Data Resources, Journal of Information \& Software Technology, 43, 1, July 2001.

[9] Davis K. Paul, Kulick Jonathan, and Egner Michael, Implications of Modern Decision Science for Military Decision-Support Systems, Santa Monica: RAND Corporation, 2005.

[10] Général Loup Francart, La maîtrise de l'information, Doctrines, 2000.

[11] Grund C.G., Meier M.C., Towards Game-based Management Decision Support: Using Serious Games to Improve the Decision Process, 2016.

[12] Leifler Ola, Affordances and Constraints of Intelligent Decision Support for Military Command and Control - Three Case Studies of Support Systems, Linköping University, 2011.

[13] Petrov V. Plamen, Zhu Qiuming, Hicks D. Jeffrey, and Stoyen D. Alexander, A Hierarchical Collective Agents Network for Real-time Sensor Fusion and Decision Support", in AAAI Workshop Technical Report WS-02-15, Menlo Park: AAAI Press, 2002.

[14] Russel, Norvig, Artificial intelligence - A modern approach, Prentice Hall, 1995.

[15] Tolk Andreas, Decision Support Systems - Technical Prerequisites and Military Requirements, in Command and Control Research and Technology Symposium Proceeding, June 2000, available at http://arxiv.org. 